\title{
Hepatitis A and E: not to be forgotten
}

\author{
Rana J. Asghar ${ }^{1}$
}

The World Health Organization (WHO) estimates that every year hepatitis A virus (HAV) infection causes nearly 1.4 million new cases worldwide and the hepatitis E virus (HEV) is responsible for 20 million new infections and over 3 million acute cases. Although in most cases HAV and HEV infections are self-limiting, HAV is estimated to kill 100000 people each year [1] and HEV nearly 60000 people annually [2]. Pregnant women are at risk of more severe disease, obstetric complications and increased mortality if infected in the third trimester of pregnancy. The faecal-oral route is a well-established mode of transmission for both HAV and $\mathrm{HEV}$ and in the case of HEV infection person-to-person transmission is also an important factor in sporadic cases [3]. Outbreaks of HAV and HEV are therefore manifestations of the poor sanitation practices and lack of clean water supplies often found in developing countries. Humanitarian crises with large refugee populations can also be fertile ground for hepatitis outbreaks alongside cholera or other waterborne outbreaks, as was recently seen among Syrian refugees in Iraq [4]. Understanding the importance of the risk of hepatitis outbreaks in displaced populations can help in identifying outbreaks quickly and responding to them in a timely manner to reduce mortality and morbidity.

HAV has 7 genotypes, with little variation in their clinical expression. HEV has 4 genotypes with quite different clinical expressions, responsible for different disease manifestations across developing and developed countries. In developing countries genotype 1 is largely responsible for outbreaks and sporadic cases, via contamination of water and the fecal-oral route. Exceptions include Mexico in South America and countries in Africa, where genotype 2 is more common [5]. Neither HAV nor HEV have chronic states, although $\mathrm{HEV}$ is reported in immunocompromised people [6].

Acute HAV infection is often clinically indistinguishable from other causes of acute viral hepatitis, and laboratory confirmation is necessary. Diagnosis of HEV has its own challenges, which may result in an underestimation of the disease burden [6]. HAV has a very effective vaccine available, and the first vaccine for $\mathrm{HEV}$ was approved in China in December 2011, although it is not yet used in any other countries. Whereas HAV seroprevalence increases with age and comes close to $100 \%$ in highly endemic countries by the age of 5 years, HEV seroprevalence tends to stay between 5\%-60\% [7].

In 2010, the WHO World Health Assembly adopted resolution WHA63.18, which called for the prevention and control of viral hepatitis, with a focus on HBV and HCV [8]. This resolution came after Member States and WHO understood the gravity of spread of viral hepatitis. In 2012, on the occasion of World Hepatitis Day, Dr Ala Alwan, WHO Regional Director for the Eastern Mediterranean, urged all stakeholders to combine their efforts to confront and combat this silent epidemic of hepatitis.

Many countries of the Eastern Mediterranean Region (EMR) are currently going through major social and political upheavals. With active or proxy wars engulfing many countries in the Region, the public health structures are under stress, creating ideal conditions for the spread of all infectious diseases, especially those spread by contaminated water and lack of sanitation services. Except for a few published articles and outbreak reports, however, very limited data are available about the prevalence of HAV and HEV from these countries or from the Region in general [9-12]. One major reason for this knowledge gap is that we are not looking for the evidence. In most developing countries, including most of those of the EMR, $\mathrm{HEV}$ is not routinely considered when a physician asks for investigations into a suspected case of viral hepatitis. Added to the diagnostic challenges is the fact that a majority of hepatitis-infected persons do not develop an acute condition that requires major health care intervention. HAV is in the same class, with few symptomatic cases reaching health-care settings, and as it is self-limiting in most cases, it is neither investigated nor reported to surveillance systems as HAV. Physicians also do not consider HAV or HEV to be serious illnesses, even though fulminant hepatitis, hepatic failure and death can occur from both infections.

More than $40 \%$ of the population of the EMR lives in just 2 countries, Egypt and Pakistan, both of which are considered endemic for HBC and HCV [8]. In Pakistan, the Field Epidemiology Laboratory Training Programme is collecting information about acute viral hepatitis cases via 5 sentinel sites throughout the country [13]. According to reports from the Pakistan viral hepatitis surveillance system HAV_responsible for more 
than $19 \%$ of all new infections - ranks second to HCV, while HEV_causing $12 \%$ of all newly diagnosed/acute cases of viral hepatitis - ranks third [14]. In Egypt, the importance of HAV has also been demonstrated through the local viral hepatitis sentinel system [15].

The lack of reliable data from the Region is preventing knowledge of the full extent of the burden of HAV and HEV in countries of the EMR. This in turn limits the ability of health planners to demonstrate to decision-makers the extent of the actual disease burden. The outcome is that fewer resources are prioritized for hepatitis control in EMR. Although our Region has all the precursors for the spread of these 2 types of hepatitis, we seem unable to better prepare ourselves to protect our populations. Awareness of HAV and HEV as major health challenges will be the first step towards effective hepatitis control strategies. Improved sanitation, provision of clean water and instigating vaccination where required could be the simple way to control both diseases. But sometimes simple is not easily accessible.

\section{References}

1. Lozano R et al. Global and regional mortality from 235 causes of death for 20 age groups in 1990 and 2010: a systematic analysis for the Global Burden of Disease Study 2010. Lancet, 2012, 380:2095-2128.

2. Hepatitis. World Health Organization Regional Office for the Eastern Mediterranean [online factsheet] (http://www.emro. who.int/health-topics/hepatitis/, accessed 13 November 2013).

3. Teshale EH, Hu DJ, Holmberg SD. The two faces of hepatitis $E$ virus. Clinical Infectious Diseases, 2010, 51:328-334.

4. WHO responds effectively to an outbreak of hepatitis A among Syrian refugees in northern Iraq. WHO Regional Office for the Eastern Mediterranean [website] (http://www.emro.who. int/irq/iraq-news/hepatitis-outbreak-2013.html, accessed 4 December 2013).

5. Hoofnagle JH, Nelson KE, Purcell RH. Hepatitis E. New England Journal of Medicine, 2012, 367:1237-1244.

6. Kamar N et al. Hepatitis E. Lancet, 2012, 379:2477-2488.

7. Gaetano S, Vicenzina F. Chapter 9. Hepatitis E. In: Serviddio G, ed. Practical management of chronic viral hepatitis. Rijeka, Croatia, InTech, 2013 [e-book] (http://dx.doi.org/10.5772/55568, accessed 4 December 2013).

8. Technical paper. The growing threats of hepatitis B and C in the Eastern Mediterranean Region: a call for action. Agenda item 6 (a). In: Fifty-sixth Session of the Regional Committee for the Eastern Mediterranean. Cairo, WHO Regional Office for the Eastern Mediterranean, 2009 (EM/RC56/3) (http://applica-
tions.emro.who.int/docs/EM_RC56_3_en.pdf, accessed 13 November 2013).

9. Hepatitis E outbreak continues in South Sudan. Weekly Epidemiological Monitor, 2013, 6(7) (http://applications.emro.who. int/dsaf/epi/2013/Epi_Monitor_2013_6_6.pdf, accessed 4 December 2013).

10. Guthmann J et al. A large outbreak of hepatitis E among a displaced population in Darfur, Sudan, 2004: the role of water treatment methods. Clinical Infectious Diseases, 2006, 42(12):1685-1691.

11. Ataei B et al. Hepatitis E virus in Isfahan province: a populationbased study. International Journal of Infectious Diseases, 2009, 13:67-71.

12. Bashir K, Hussain N, Hasnain ES. Seroprevalence of hepatitis E virus immunoglobulin $\mathrm{G}$ and $\mathrm{M}$ antibodies in adults: a hospitalbased study. Indian Journal of Medical Microbiology, 2009, 27:139-141.

13. Establishment of a Viral Hepatitis Surveillance System-Pakistan, 2009-2011. Morbidity and Mortality Weekly Report, 2011, 60:1385-1390.

14. Acute viral hepatitis sentinel surveillance. Monthly report: March 2013. Islamabad, Pakistan Field Epidemiology and Laboratory Training Program, 2013.

15. Talaat $M$ et al. Sentinel surveillance for patients with acute hepatitis in Egypt, 2001-04. Eastern Mediterranean Health Journal, 2010, 16:134-140. 